\title{
ASYMPTOTIC STABILITY OF LINEAR CONSERVATIVE SYSTEMS WHEN COUPLED WITH DIFFUSIVE SYSTEMS
}

\author{
Denis Matignon ${ }^{1}$ And Christophe Prieur ${ }^{2}$
}

\begin{abstract}
In this paper we study linear conservative systems of finite dimension coupled with an infinite dimensional system of diffusive type. Computing the time-derivative of an appropriate energy functional along the solutions helps us to prove the well-posedness of the system and a stability property. But in order to prove asymptotic stability we need to apply a sufficient spectral condition. We also illustrate the sharpness of this condition by exhibiting some systems for which we do not have the asymptotic property.
\end{abstract}

Mathematics Subject Classification. 35B37, 93C20, 93D20.

Received March 16, 2004. Revised October 21, 2004.

\section{INTRODUCTION}

In this paper we study an oscillator damped by a positive pseudo-differential operator of diffusive type. This model represents the wave equation when viscous and thermal losses are concentrated in a boundary layer near the walls of the duct; averaging the acoustical quantities on the cross-section gives rise to a $\partial_{t}^{\frac{1}{2}}$ equivalent operator (see [3, 21]), which also appears in other acoustical contexts, see e.g. [9]. This model has been introduced earlier in [14,15] restated in the half-line in [8] (see also [11] for a new derivation of this equation). Taking the projection on one mode of the vibrating structure, we arrive at the model under study. The pseudo-differential operators of diffusive type under consideration in this paper belong to the class of causal pseudo-differential operators (in the time variable) which have a simple diagonal realization in infinite dimension together with interesting energy balance and dissipativity properties.

We use this energy balance to prove the existence and uniqueness of solutions, and to check that the energy of the oscillator, in closed-loop with the pseudo-differential operator, decreases (see also [10] for a more general well-posedness problem with fractional derivatives). As the energy is minimal at the equilibrium, a possible guess is the stability property. But, applying LaSalle's invariance principle requires a precompactness property (see e.g. $[4,5]$ ), which is not easy to get a priori. More precisely, a sufficient compactness criterion, such as [16], Theorem 3.65, does not apply in our cases, because of the unboundedness of the $\xi$-domain for the diffusion equation.

Keywords and phrases. Asymptotic stability, well-posed systems, Lyapunov functional, diffusive representation, fractional calculus.

1 Télécom Paris, dépt TSI \& CNRS, UMR 5141, 37-39 rue Dareau, 75014 Paris, France; matignon@tsi.enst.fr

2 LAAS - CNRS, 7 avenue du Colonel Roche 31077 Toulouse, France; prieur@laas.fr

(c) EDP Sciences, SMAI 2005 
Therefore in order to prove the asymptotic stability, we resort to the stability theorem given in [1]. To be self-contained, let us recall briefly the statement of this result:

Theorem 1.1 [1], Stability theorem). Let us consider the infinitesimal generator $\mathcal{A}$ of a bounded $\mathcal{C}^{0}$-semigroup on a reflexive Banach space. Assume that no eigenvalue of $\mathcal{A}$ lies on the imaginary axis. If $\sigma(\mathcal{A}) \cap i \mathbb{R}$ is countable, then the semigroup generated by $\mathcal{A}$ is asymptotically stable, which means that the solutions of the differential equation $x^{\prime}(t)=\mathcal{A} x(t)$ tend to 0 with $t \rightarrow \infty$.

In this paper we use this stability theorem. Note that, in our case, this stability theorem is equivalent to [17], since we work with Hilbert spaces which are reflexive Banach spaces. Moreover we can not use earlier stability theorems such as Tauberian theorem given in $[12,13,20]$. Indeed, in our case, $\sigma(\mathcal{A}) \cap i \mathbb{R}$ is not empty. See also the comparaison of these results in [1].

Two classes of diffusive pseudo-differential operators will be treated separately depending on their realization: in Section 2 we consider standard diffusive operators, such as fractional integrals $\partial_{t}^{-\alpha}$, with $\alpha \in \mathbb{R}_{+}$and $0<\alpha<1$, with negative asymptotic order; whereas, in Section 3, we consider extended diffusive operators, such as fractional derivatives $\partial_{t}^{\beta}$, with $\beta \in \mathbb{R}_{+}$and $0<\beta<1$, with positive asymptotic order. Since the functional spaces used for the realizations of standard or extended diffusive systems happen to be different, we need to study them separately.

More precisely we study in this paper, systems of the form

$$
\ddot{\theta}+a\left(\frac{\mathrm{d}}{\mathrm{d} t} \mathcal{D}_{\nu}\right)(\dot{\theta})+b \mathcal{D}_{\mu}(\dot{\theta})+c \theta=u_{e}(t),
$$

where $\theta:[0,+\infty) \rightarrow \mathbb{C}$ is an unknown function, $\mathcal{D}_{\mu}$ and $\mathcal{D}_{\nu}$ are two causal pseudo-differential operators of diffusive type with measure $\mu$ and $\nu$ respectively. We assume moreover that $a, b$ and $c$ are three non-negative real numbers, with $a b \neq 0$, and $u_{e}$ is an external input vanishing for sufficiently large time. The closed-loop system we get is, for all $(\xi, t) \in[0,+\infty)^{2}$,

$$
\partial_{t}\left(\begin{array}{c}
\theta(t) \\
\omega(t) \\
\Phi(\xi, t) \\
\tilde{\Phi}(\xi, t)
\end{array}\right)=\left(\begin{array}{c}
\omega \\
-a \int_{0}^{\infty}(\omega(t)-\xi \tilde{\Phi}(\xi, t)) \mathrm{d} \nu(\xi)-b \int_{0}^{\infty} \Phi(\xi, t) \mathrm{d} \mu(\xi)-c \theta(t)+u_{e}(t) \\
-\xi \Phi(\xi, t)+\omega(t) \\
-\xi \tilde{\Phi}(\xi, t)+\omega(t) \\
\Phi(\xi, 0)=0 \\
\tilde{\Phi}(\xi, 0)=0
\end{array}\right.
$$

Assuming that

$$
\int_{0}^{\infty} \frac{1}{1+\xi} \mathrm{d} \mu(\xi)<\infty
$$

and

$$
\int_{0}^{\infty} \frac{1}{1+\xi} \mathrm{d} \nu(\xi)<\infty,
$$

using infinite-dimensional realizations of the operators $\mathcal{D}_{\mu}$ and $\frac{\mathrm{d}}{\mathrm{d} t} \mathcal{D}_{\nu}$, we state $\Phi$ and $\tilde{\Phi}$ on appropriate Hilbert spaces i.e. $H:=L^{2}([0,+\infty), \mathrm{d} \mu(\xi))$ and $\tilde{H}:=L^{2}([0,+\infty), \xi \mathrm{d} \nu(\xi))$ respectively, and using [1], Stability theorem, we prove

Theorem 1.2. Let $\mu$ be a positive non-null measure satisfying (3) and such that $\mu(\{0\})=0$ and $\nu$ be a positive non-null measure satisfying (4). Let $p \geq 1$. Let $u_{e} \in L^{p}([0,+\infty), \mathrm{d} t)$ be such that $u_{e}(t)=0$ for sufficiently large time. 
Then, we have the asymptotic stability property for (2) i.e., for all $\left(\theta_{0}, \omega_{0}\right) \in \mathbb{C}^{2}$, the solution of (2) with initial condition $\left(\theta_{0}, \omega_{0}, 0,0\right)$ at $t=0$ satisfies

$$
(\theta, \omega, \Phi, \tilde{\Phi})(t) \rightarrow_{t \rightarrow \infty} 0 \text { in } \mathbb{C}^{2} \times H \times \tilde{H} .
$$

Moreover we prove that this theorem is sharp, more precisely we prove by exhibiting some examples that the assumption $\mu(\{0\})=0$ cannot be omitted and it is possible to get different qualitative behaviors. Moreover we note that non-exponential stability may occur.

Note that system (2) has already been studied in [7] but without a proof of asymptotic stability. Diffusive systems have been first introduced in [22], Section 5, and independently in [19]. More generally conservative linear systems have been studied in several papers. See e.g. [23-25] where the energy balance is a key-point as in our paper.

The paper is organized as follows. First in Section 2 we study an oscillator coupled with an infinitedimensional system of standard diffusive type and we prove the asymptotic property when it is true. In Section 3, we study an oscillator coupled with a system of extended diffusive type. We state the main theorem, i.e. the case of a diffusive system of both types in Section 4. Section 5 summarizes the main contributions of the work and points to some open problems and future research directions.

\section{An oscillator COUPled With a STANDARD DifFusive System}

In this section we study an oscillator damped by a pseudo-differential operator with a diffusive representation. Fractional integrals $\partial_{t}^{-\alpha}$, with $0<\alpha<1$, with negative asymptotic order, are a special case of such standard diffusive operators. We prove, when it is true, the asymptotic stability property in this case.

Let us first state the system under consideration in this section.

\subsection{Statement of the problem}

We study the following second-order mono-dimensional linear differential equation:

$$
\ddot{\theta}+\mathcal{D}_{\mu}(\dot{\theta})+c \theta=u_{e}(t)
$$

where $\theta:[0,+\infty) \rightarrow \mathbb{C}$ is an unknown function, $\mathcal{D}_{\mu}$ is a diffusive pseudo-differential operator with measure $\mu, c$ is a non-negative real number and $u_{e}$ is an external input vanishing for sufficiently large time, i.e. we suppose that there exists $T_{e}>0$ such that

$$
u_{e}(t)=0, \forall t \geq T_{e} .
$$

In all the following we assume that the measure $\mu$ is positive, non-null and satisfies

$$
c_{\mu}:=\int_{0}^{\infty} \frac{1}{1+\xi} \mathrm{d} \mu(\xi)<\infty .
$$

Let us consider the realization of the input-output operator $\mathcal{D}_{\mu}$ in the observator form (see e.g. [19])

$$
\left\{\begin{aligned}
\partial_{t} \Phi(\xi, t) & =-\xi \Phi(\xi, t)+u(t), \forall \xi \geq 0 \\
\Phi(., 0) & =0 \\
y(t) & :=\mathcal{D}_{\mu}(u)=\int_{0}^{\infty} \Phi(\xi, t) \mathrm{d} \mu(\xi) \\
u(t) & :=\dot{\theta}(t)
\end{aligned}\right.
$$

where $\Phi:[0,+\infty) \times[0,+\infty) \rightarrow \mathbb{C}$ is the state of $(8), u:[0,+\infty) \rightarrow \mathbb{C}$ is the input and $y:[0,+\infty) \rightarrow \mathbb{C}$ the observation. 
Let us recall the functional spaces where this realization makes sense. To do this let us introduce, for $k \in \mathbb{N}$, the subspace $H^{k}$ of $L_{\mathrm{loc}}^{2}\left(\mathbb{R}^{+}, \mathrm{d} \mu\right)$, defined by

$$
H^{k}=\left\{\phi \in L_{\mathrm{loc}}^{2}\left(\mathbb{R}^{+}, \mathrm{d} \mu\right), \int_{0}^{\infty}(1+\xi)^{k}|\phi(\xi)|^{2} \mathrm{~d} \mu(\xi)<\infty\right\}
$$

and denote $V=H^{1}, H=H^{0}$. We equip $H$ with the following hermitian product, for all $\phi$ and $f$ in $H$,

$$
\langle\phi, f\rangle_{H}=\int_{0}^{\infty} \phi(\xi) \overline{f(\xi)} \mathrm{d} \mu(\xi) .
$$

By identification of $H$ with its dual, we have $H^{1}=V \hookrightarrow H \hookrightarrow V^{\prime}=H^{-1}$ with continuous and dense embeddings.

Let us now introduce the linear operators defining the differential system (5) and (8).

\subsection{An abstract formulation}

Let us now introduce the linear operator defining (5) as a conservative ordinary differential operator in closed-loop with the pseudo-differential operator (8):

$$
\partial_{t}\left(\begin{array}{c}
\theta \\
\omega \\
\Phi
\end{array}\right)=\mathcal{A}\left(\begin{array}{c}
\theta \\
\omega \\
\Phi
\end{array}\right)+\mathcal{B} u_{e}:=\left(\begin{array}{c}
\omega \\
-c \theta-\int_{0}^{\infty} \Phi \mathrm{d} \mu \\
\omega-\xi \Phi
\end{array}\right)+\left(\begin{array}{c}
0 \\
u_{e} \\
0
\end{array}\right)
$$

with $\mathcal{A}: \mathcal{D}(\mathcal{A}) \rightarrow \mathbb{C}^{2} \times H$ and $\mathcal{B}: \mathbb{C} \rightarrow \mathbb{C}^{2} \times H$ two suitable linear operators and

$$
\mathcal{D}(\mathcal{A})=\left\{(\theta, \omega, \phi) \in \mathbb{C}^{2} \times V \text {, s.t. } \xi \mapsto \omega-\xi \phi(\xi) \in H\right\} .
$$

We equip $\mathbb{C}^{2} \times H$ with the following hermitian product, for all $(\theta, \omega, \phi)$ and $(x, y, f)$ in $\mathbb{C}^{2} \times H$,

$$
\left\langle\left(\begin{array}{l}
\theta \\
\omega \\
\phi
\end{array}\right),\left(\begin{array}{l}
x \\
y \\
f
\end{array}\right)\right\rangle_{\mathbb{C}^{2} \times H}=c \theta \bar{x}+\omega \bar{y}+\int_{0}^{\infty} \phi(\xi) \overline{f(\xi)} \mathrm{d} \mu(\xi) .
$$

Let $\mathcal{E}: \mathbb{C}^{2} \times H \rightarrow \mathbb{R}$ be defined, for all $(\theta, \omega, \phi) \in \mathbb{C}^{2} \times H$, by

$$
\mathcal{E}(\theta, \omega, \phi)=\frac{1}{2}\left(\|(\theta, \omega, \phi)\|_{\mathbb{C}^{2} \times H}\right)^{2} .
$$

Let us compute, at least formally the time-derivative of the function $\mathcal{E}$, along the solutions of (9) (it will make sense in Sect. 2.3 below):

$$
\begin{aligned}
\frac{\mathrm{d}}{\mathrm{d} t} \mathcal{E}(\theta, \dot{\theta}, \Phi) & =\Re(\dot{\theta} \overline{(\ddot{\theta}+c \theta)})+\Re\left(\int_{0}^{\infty} \Phi \overline{\partial_{t} \Phi} \mathrm{d} \mu(\xi)\right), \\
& =\Re\left(\dot{\theta} \overline{u_{e}(t)}\right)-\int_{0}^{\infty} \xi|\Phi|^{2} \mathrm{~d} \mu(\xi)
\end{aligned}
$$

where $\Re$ denotes the real part of a complex number. Thus, for sufficiently large time, we have $\frac{\mathrm{d}}{\mathrm{d} t} \mathcal{E} \leq 0$ (recall (6)). This allows us to detect the asymptotic stability property which is proved, when it is true, in Section 2.4. But let us prove first an existence result. 


\subsection{Existence and uniqueness of the solutions}

We prove the existence and uniqueness results of the (classical or weak) solutions of (9) using the classical theory of semi-groups (see e.g. [6]). To do this let us start by proving the following:

Lemma 2.1. The operator $-\mathcal{A}$ is monotone.

Proof. Due to (9) we have, for all $(\theta, \omega, \phi) \in \mathcal{D}(\mathcal{A})$,

$$
\begin{aligned}
\Re\left\langle-\mathcal{A}\left(\begin{array}{c}
\theta \\
\omega \\
\phi
\end{array}\right),\left(\begin{array}{l}
\theta \\
\omega \\
\phi
\end{array}\right)\right\rangle_{\mathbb{R}^{2} \times H} & =\Re\left\langle\left(\begin{array}{c}
-\omega \\
c \theta+\int_{0}^{\infty} \phi \mathrm{d} \mu \\
-\omega+\xi \phi
\end{array}\right),\left(\begin{array}{l}
\theta \\
\omega \\
\phi
\end{array}\right)\right\rangle_{\mathbb{C}^{2} \times H} \\
& =\Re(-c \bar{\theta} \omega+c \theta \bar{\omega})+\Re\left(\bar{\omega} \int_{0}^{\infty} \phi \mathrm{d} \mu-\int_{0}^{\infty} \omega \bar{\phi} \mathrm{d} \mu\right)+\Re\left(\int_{0}^{\infty} \xi|\phi|^{2} \mathrm{~d} \mu\right) \\
& =\int_{0}^{\infty} \xi|\phi|^{2} \mathrm{~d} \mu \geq 0 .
\end{aligned}
$$

We have now to prove the maximality of the operator $-\mathcal{A}$. This proof is quite standard, but still, when solving the resolvent equation:

$$
(I-\mathcal{A})(\theta, \omega, \phi)^{\prime}=(x, y, f)^{\prime},
$$

special care must be taken to prove that the solution does belong to the appropriate functional space, namely $\mathcal{D}(\mathcal{A})$.

Lemma 2.2. The operator $-\mathcal{A}$ is maximal.

Proof. This lemma is a special case of Proposition A.2 given in Appendix with $\lambda=1$.

Since $-\mathcal{A}$ is monotone and maximal and using [2], Chapter 7 , we get that, for all $\left(\theta_{0}, \omega_{0}, \phi_{0}\right) \in \mathcal{D}(\mathcal{A})$, there exists a unique $(\theta, \omega, \Phi) \in \mathcal{C}^{1}\left([0,+\infty), \mathbb{C}^{2} \times H\right) \cap \mathcal{C}^{0}([0,+\infty), \mathcal{D}(\mathcal{A}))$ such that

$$
\partial_{t}(\theta, \omega, \Phi)^{\prime}=\mathcal{A}(\theta, \omega, \Phi)^{\prime},
$$

with the initial condition

$$
(\theta, \omega, \Phi)^{\prime}(t=0)=\left(\theta_{0}, \omega_{0}, \phi_{0}\right)^{\prime} .
$$

Thus the formal computation of (11)-(12) makes sense with $u_{e}=0$ and

$$
\frac{\mathrm{d}}{\mathrm{d} t}\|\theta(t), \omega(t), \Phi(., t)\|_{\mathbb{C}^{2} \times V} \leq 0 .
$$

Integrating this inequality gives

$$
\left\|(\theta(t), \omega(t), \Phi(., t))^{\prime}\right\|_{\mathbb{C}^{2} \times H} \leq\left\|\left(\theta_{0}, \omega_{0}, \phi_{0}\right)^{\prime}\right\|_{\mathbb{C}^{2} \times H},
$$

meaning exactly that the $\mathcal{C}^{0}$-semigroup generated by $\mathcal{A}$ is a contraction semigroup.

Moreover, by applying e.g. [6], Theorem 3.1.3, we have, for all $u_{e} \in \mathcal{C}^{1}([0,+\infty), \mathbb{C})$, and for all $\left(\theta_{0}, \theta_{1}\right) \in \mathbb{C}^{2}$, the existence of a unique classical solution of $(9)$ defined on $[0,+\infty)$ with the initial condition

$$
\theta(0)=\theta_{0}, \quad \omega(0)=\omega_{0}, \quad \Phi(\xi, 0)=0, \forall \xi \in[0,+\infty) .
$$

Thus the formal computations of (11) and (12) make sense and, thanks to (6), the function $\mathcal{E}$ is non increasing for $t$ large enough. 
Let us now note that, for all $p \geq 1$, for all $u_{e} \in L^{p}([0,+\infty), \mathrm{d} t)$ and for all $\left(\theta_{0}, \theta_{1}\right) \in \mathbb{C}^{2}$, thanks to [6], Theorem 3.1.7, there exists a unique weak solution of $(9)$ defined on $[0,+\infty)$ with the initial condition (16).

In all the following we will consider only weak solutions.

With this existence and uniqueness result at hand, let us look for the asymptotic behavior of solutions of (9).

\subsection{Asymptotic stability}

In this section, we use, when it is possible, a Tauberian theorem to prove the asymptotic stability property of the system (9). We also state the main result of this section.

To the best of our knowledge, we can not apply LaSalle's principle with the function $\mathcal{E}$ defined by (10) as a Lyapunov function. The main difficulty to use LaSalle's principle is the precompactness of the solutions of (9) which is not clear to get a priori.

Let us find the conditions for which [1], Stability theorem, applies.

It was already checked in Section 2.3 that $\mathcal{A}$ generates a bounded $\mathcal{C}^{0}$-semigroup.

Let us now prove

Lemma 2.3. We have

$$
\sigma(\mathcal{A}) \cap\{i \alpha, \alpha \in \mathbb{R}, \alpha \neq 0\}=\emptyset .
$$

Proof. This proof is a special case of Proposition A.2 with $\lambda=i \alpha, \alpha \in \mathbb{R}, \alpha \neq 0$.

Thanks to Lemma $2.3, \sigma(\mathcal{A}) \cap i \mathbb{R}$ is at most countable as required by the necessary conditions of [1], Stability theorem.

Let us prove the following

Lemma 2.4. $\lambda=0$ is an eigenvalue of $\mathcal{A}$ if and only if $\mu(\{0\}) \neq 0$.

Proof. From $(9)$ we get that $(\theta, \omega, \phi) \in \operatorname{Ker}(\mathcal{A})$ if and only if

$$
\left\{\begin{aligned}
\omega & =0 \\
c \theta+\int_{0}^{\infty} \phi \mathrm{d} \mu & =0 \\
\omega-\xi \phi & =0, \text { for } \mu \text {-almost every } \xi \text { in }[0, \infty)
\end{aligned}\right.
$$

which is equivalent to

$$
\left\{\begin{array}{l}
\omega=0 \\
\phi=0, \text { for } \mu \text {-almost every } \xi \text { in }(0, \infty) \\
\theta=-\frac{\mu(\{0\})}{c} \phi(0) .
\end{array}\right.
$$

Thus we get the equivalence stated in Lemma 2.4.

Remark 2.5. If $\mu(\{0\}) \neq 0$, we get from the proof of Lemma 2.4 that the kernel of $\mathcal{A}$ is

$$
\mathbb{C}\left(-\mu(\{0\}), 0, \phi_{0}\right)
$$

where $\phi_{0}:[0, \infty) \rightarrow \mathbb{C}$ is equal to 0 , for $\xi>0$ and equal to $c$ for $\xi=0$. This remark will be useful below.

From Lemma 2.3, Lemma 2.4 and [1], Stability theorem, we get, the asymptotic stability of (5) in closed-loop with (8):

Theorem 2.6. Let $\mu$ be a positive non-null measure satisfying (7) and such that $\mu(\{0\})=0$. Let $p \geq 1$. Let $u_{e} \in L^{p}([0,+\infty), \mathrm{d} t)$ be such that $u_{e}(t)=0$ for sufficiently large time. 
Then, we have the asymptotically stability property, i.e. for all $\left(\theta_{0}, \omega_{0}\right) \in \mathbb{C}^{2}$, the solution of (5) in closed-loop with (8), with initial condition $\left(\theta_{0}, \omega_{0}, 0\right)$ at $t=0$, satisfies

$$
\mathcal{E}(\theta, \dot{\theta}, \Phi)(t) \rightarrow{ }_{t \rightarrow \infty} 0
$$

or equivalently $(\theta, \dot{\theta}, \Phi)(t) \rightarrow_{t \rightarrow \infty} 0$ in $\mathbb{C}^{2} \times H$.

Remark 2.7. The conclusion of Theorem 2.6 is sharp in the sense that we can have a non-exponential stability. See e.g. the case $\mathrm{d} \mu_{\alpha}(\xi)=\frac{\sin (\pi \alpha)}{\pi} \frac{1}{\xi^{\alpha}} \mathrm{d} \xi$, for all $\xi>0$ and fractional differential equations with a decay which is not of exponential type. See [18], example 2.4.2, together with [18], Theorem 2.17.

Remark 2.8. Now, when $\mu(\{0\})=C_{0}>0$, thanks to Lemma $2.4, \lambda=0$ is an eigenvalue of $\mathcal{A}$, hence the system can not be asymptotically stable. Nevertheless, the influence of the constant $C_{0}$ on the subsystem $(\theta, \omega)$ can be very different. For example,

- $C_{0}$ can change the frequency of oscillations and their amplitude, in the case $\mu=C_{0} \delta_{0}$ where $\delta_{0}$ is the Dirac measure at $\xi=0$;

- $C_{0}$ can also create a non-zero limit state in the case $\mu=C_{0}+\mu_{\alpha}$ where $\mathrm{d} \mu_{\alpha}(\xi)=\frac{\sin (\pi \alpha)}{\pi} \frac{1}{\xi^{\alpha}} \mathrm{d} \xi$, for all $\xi>0$.

The full proof of this remark is given in Appendix B, by studying both these examples.

\section{An OSCILlator COUPLED WITH AN EXTENDED DIFFUSIVE SYSTEM}

In this section we study an oscillator damped by a pseudo-differential operator with an extended diffusive representation. Fractional derivatives $\partial_{t}^{\beta}$, with $0<\beta<1$, with positive asymptotic order $\beta$ are a special case of such extended diffusive operators. We prove, when it is true, the asymptotic stability property in this case.

Let us first state the system under consideration in this section.

\subsection{Statement of the problem}

We study the following second-order mono-dimensional linear differential equation:

$$
\ddot{\theta}+\left(\frac{\mathrm{d}}{\mathrm{d} t} \mathcal{D}_{\nu}\right)(\dot{\theta})+c \theta=u_{e}(t)
$$

where $\theta:[0,+\infty) \rightarrow \mathbb{C}$ is an unknown function, $\mathcal{D}_{\nu}$ is a diffusive pseudo-differential operator with measure $\nu$ (as already introduced in Sect. 2), $c$ is a non-negative real number and $u_{e}$ is an external input vanishing for sufficiently large time, i.e. we suppose that there exists $T_{e}>0$ such that

$$
u_{e}(t)=0, \forall t \geq T_{e} .
$$

In all the following we assume that the measure $\nu$ is positive, non-null and satisfies:

$$
c_{\nu}:=\int_{0}^{\infty} \frac{1}{1+\xi} \mathrm{d} \nu(\xi)<\infty .
$$

Let us consider the realization of the input-output operator $\frac{\mathrm{d}}{\mathrm{d} t} \mathcal{D}_{\nu}$ in the observator form

$$
\left\{\begin{aligned}
\partial_{t} \tilde{\Phi} & =-\xi \tilde{\Phi}+u(t), \forall \xi \geq 0 \\
\tilde{\Phi}(., 0) & =0 \\
z(t) & :=\frac{\mathrm{d}}{\mathrm{d} t} \mathcal{D}_{\nu}(u)=\int_{0}^{\infty} \partial_{t} \tilde{\Phi}(\xi, t) \mathrm{d} \nu(\xi) \\
u(t) & :=\dot{\theta}(t)
\end{aligned}\right.
$$


where $\tilde{\Phi}:[0,+\infty) \times[0,+\infty) \rightarrow \mathbb{C}$ is the state of $(21), u:[0,+\infty) \rightarrow \mathbb{C}$ is the input and $z:[0,+\infty) \rightarrow \mathbb{C}$ the observation.

Let us introduce the following subpaces of $L_{\mathrm{loc}}^{2}\left(\mathbb{R}^{+}, \mathrm{d} \nu\right)$ :

$$
\begin{aligned}
& V=\left\{\tilde{\phi} \in L_{\mathrm{loc}}^{2}\left(\mathbb{R}^{+}, \mathrm{d} \nu\right), \int_{0}^{\infty}(1+\xi)|\tilde{\phi}|^{2} \mathrm{~d} \nu(\xi)<\infty\right\}, \\
& \tilde{H}=\left\{\tilde{\phi} \in L_{\mathrm{loc}}^{2}\left(\mathbb{R}^{+}, \mathrm{d} \nu\right), \int_{0}^{\infty} \xi|\tilde{\phi}|^{2} \mathrm{~d} \nu(\xi)<\infty\right\} .
\end{aligned}
$$

We equip $\tilde{H}$ with the following hermitian product, for all $\tilde{\phi}$ and $\tilde{f}$ in $\tilde{H}$,

$$
\langle\tilde{\phi}, \tilde{f}\rangle_{\tilde{H}}=\int_{0}^{\infty} \xi \tilde{\phi}(\xi) \overline{\tilde{f}(\xi)} \mathrm{d} \nu(\xi)
$$

Let us now write down the linear operators defining the differential system (18) and (21).

\subsection{An abstract formulation}

Let us now introduce the linear operator defining (18) as a conservative ordinary differential operator in closed-loop with the pseudo-differential operator (21):

$$
\partial_{t}\left(\begin{array}{c}
\theta \\
\omega \\
\tilde{\Phi}
\end{array}\right)=\tilde{\mathcal{A}}\left(\begin{array}{c}
\theta \\
\omega \\
\tilde{\Phi}
\end{array}\right)+\tilde{\mathcal{B}} u_{e}:=\left(\begin{array}{c}
\omega \\
-c \theta-\int_{0}^{\infty}(\omega-\xi \tilde{\Phi}) \mathrm{d} \nu \\
\omega-\xi \tilde{\Phi}
\end{array}\right)+\left(\begin{array}{c}
0 \\
u_{e} \\
0
\end{array}\right)
$$

with $\tilde{\mathcal{A}}: \mathcal{D}(\tilde{\mathcal{A}}) \rightarrow \mathbb{C}^{2} \times \tilde{H}$ and $\tilde{\mathcal{B}}: \mathbb{C} \rightarrow \mathbb{C}^{2} \times \tilde{H}$ two suitable linear operators and

$$
\mathcal{D}(\tilde{\mathcal{A}})=\left\{(\theta, \omega, \tilde{\phi}) \in \mathbb{C}^{2} \times \tilde{H} \text {, s.t. } \xi \mapsto \omega-\xi \tilde{\phi}(\xi) \in V\right\} .
$$

We equip $\mathbb{C}^{2} \times \tilde{H}$ with the following hermitian product, for all $(\theta, \omega, \tilde{\phi})$ and $(x, y, \tilde{f})$ in $\mathbb{C}^{2} \times \tilde{H}$,

$$
\left\langle\left(\begin{array}{c}
\theta \\
\omega \\
\tilde{\phi}
\end{array}\right),\left(\begin{array}{l}
x \\
y \\
\tilde{f}
\end{array}\right)\right\rangle_{\mathbb{C}^{2} \times \tilde{H}}=c \theta \bar{x}+\omega \bar{y}+\int_{0}^{\infty} \xi \tilde{\phi}(\xi) \overline{\tilde{f}(\xi)} \mathrm{d} \nu(\xi) .
$$

Let $\tilde{\mathcal{E}}: \mathbb{C}^{2} \times \tilde{H} \rightarrow \mathbb{R}$ be the function defined, for all $(\theta, \omega, \tilde{\phi}) \in \mathbb{C}^{2} \times \tilde{H}$, by

$$
\tilde{\mathcal{E}}(\theta, \omega, \tilde{\phi})=\frac{1}{2}\left(\|(\theta, \omega, \tilde{\phi})\|_{\mathbb{C}^{2} \times \tilde{H}}\right)^{2} .
$$

Let us compute, at least formally, the time-derivative of the function $\tilde{\mathcal{E}}$, along the solutions of (21),

$$
\begin{aligned}
& \frac{\mathrm{d}}{\mathrm{d} t} \tilde{\mathcal{E}}(\theta, \dot{\theta}, \tilde{\Phi})=\Re\left(\dot{\theta} \overline{(\ddot{\theta}+c \theta)}+\Re\left(\int_{0}^{\infty} \xi \tilde{\Phi} \overline{\partial_{t} \tilde{\Phi}} \mathrm{d} \nu(\xi),\right.\right. \\
& =\dot{\theta} u_{e}(t)-\int_{0}^{\infty}\left|\partial_{t} \tilde{\Phi}\right|^{2} \mathrm{~d} \nu(\xi) .
\end{aligned}
$$

Thus, for sufficiently large time, we have $\frac{\mathrm{d}}{\mathrm{d} t} \tilde{\mathcal{E}} \leq 0$ (recall (19)). 


\subsection{Existence and uniqueness of the solutions}

We prove the existence and uniqueness results of the weak solutions of (21) using classical theory on semigroups. To do this let us start by proving the following:

Lemma 3.1. The operator $-\tilde{\mathcal{A}}$ is monotone.

Proof. Due to $(22)$ we have, for all $(\theta, \omega, \tilde{\phi}) \in \mathcal{D}(\tilde{\mathcal{A}})$,

$$
\begin{aligned}
\Re\left\langle-\tilde{\mathcal{A}}\left(\begin{array}{c}
\theta \\
\omega \\
\tilde{\phi}
\end{array}\right),\left(\begin{array}{c}
\theta \\
\omega \\
\tilde{\phi}
\end{array}\right)\right\rangle_{\mathbb{C}^{2} \times \tilde{H}} & =\Re\left\langle\left(\begin{array}{c}
c \theta+\int_{0}^{\infty}(\omega-\xi \tilde{\phi}) \mathrm{d} \mu \\
-\omega+\xi \tilde{\phi}
\end{array}\right),\left(\begin{array}{c}
\theta \\
\omega \\
\tilde{\phi}
\end{array}\right)\right\rangle_{\mathbb{C}^{2} \times \tilde{H}} \\
& =\Re(-c \bar{\theta} \omega+c \theta \bar{\omega})+\Re\left(\bar{\omega} \int_{0}^{\infty}(\omega-\xi \tilde{\phi}) \mathrm{d} \nu\right)+\Re\left(\int_{0}^{\infty} \xi(-\omega+\xi \phi) \overline{\tilde{\phi}} \mathrm{d} \nu\right) \\
& =\int_{0}^{\infty}|\omega-\xi \tilde{\phi}|^{2} \mathrm{~d} \nu \geq 0 .
\end{aligned}
$$

We have now to prove the maximality of the operator $-\tilde{\mathcal{A}}$.

Lemma 3.2. The operator $-\tilde{\mathcal{A}}$ is maximal.

Proof. This lemma is a special case of Proposition A.4 given in Appendix with $\check{C}=0$ and $\lambda=1$.

Note that $\tilde{\mathcal{A}}$ generates a contraction semigroup and thanks to [6], Theorem 3.1.7, for all $p \geq 1$, for all $u \in L^{p}([0,+\infty), \mathrm{d} t)$, and for all $\left(\theta_{0}, \theta_{1}\right) \in \mathbb{C}^{2}$, we have the existence and the uniqueness of a weak solution of $(21)$ defined on $[0,+\infty)$ with initial condition

$$
\theta(0)=\theta_{0}, \quad \dot{\theta}(0)=\theta_{1}, \quad \tilde{\Phi}(\xi, 0)=0, \forall \xi \in[0,+\infty) .
$$

With this existence and uniqueness result at hand, let us look for the asymptotic stability property of solutions of $(21)$.

\subsection{Asymptotic stability}

In this section we will apply, when it is possible, [1], Stability theorem, again.

Note first that the operator $\tilde{\mathcal{A}}$ defines a bounded semigroup.

Let us now prove the following

Lemma 3.3. We have

$$
\sigma(\tilde{\mathcal{A}}) \cap\{i \alpha, \alpha \in \mathbb{R}, \alpha \neq 0\}=\emptyset
$$

Proof. This proof is a special case of Proposition A.4 with $\check{C}=0$ and $\lambda=i \alpha, \alpha \in \mathbb{R}, \alpha \neq 0$.

Thanks to Lemma $3.3, \sigma(\tilde{\mathcal{A}}) \cap i \mathbb{R}$ is at most countable as required by the necessary conditions of [1], Stability theorem.

We have to study the value $\lambda=0$ separately. Let us prove the following

Lemma 3.4. $\lambda=0$ is an eigenvalue of $\tilde{\mathcal{A}}$ if and only if $\nu(\{0\}) \neq 0$.

Proof. From $(22)$ we get that $(\theta, \omega, \tilde{\phi}) \in \operatorname{Ker}(\tilde{\mathcal{A}})$ if and only if

$$
\left\{\begin{aligned}
\omega & =0, \\
c \theta+\int_{0}^{\infty} \xi \tilde{\phi} \mathrm{d} \nu & =0, \\
\omega-\xi \tilde{\phi} & =0, \text { for } \nu \text {-almost every } \xi \text { in }[0, \infty),
\end{aligned}\right.
$$


which is equivalent to

$$
\left\{\begin{array}{l}
\omega=0, \\
\tilde{\phi}=0, \text { for } \nu \text {-almost every } \xi \text { in }(0, \infty), \\
\theta=0 .
\end{array}\right.
$$

Thus we get the equivalence stated in Lemma 3.4.

Remark 3.5. From the proof of Lemma 3.4 we deduce that, if $\nu(\{0\} \neq 0$,

$$
\operatorname{Ker}(\tilde{\mathcal{A}})=\mathbb{R}\left(0,0, \tilde{\phi}_{0}\right)
$$

with $\tilde{\phi}_{0}:[0, \infty) \rightarrow \mathbb{C}$ is equal to 0 , for all $\xi>0$ and equal to 1 for $\xi=0$.

From [1], Stability theorem, and Lemmas 3.3 and 3.4, we deduce the stability result:

Proposition 3.6. Let $\nu$ be a positive non-null measure satisfying (20) and such that $\nu(\{0\})=0$. Let $p \geq 1$. Let $u_{e} \in L^{p}([0,+\infty), \mathrm{d} t)$ be such that $u_{e}(t)=0$ for sufficiently large time.

Then we have a stability property for $\tilde{\mathcal{A}}$, i.e. for all $\theta_{0}$ and $\omega_{0}$, the solution of (18) in closed-loop with (21), with initial condition $\left(\theta_{0}, \omega_{0}, 0\right)$, satisfies

$$
\tilde{\mathcal{E}}(\theta, \dot{\theta}, \tilde{\Phi})(t) \rightarrow_{t \rightarrow \infty} 0
$$

or equivalently $(\theta, \dot{\theta}, \tilde{\Phi})(t) \rightarrow_{t \rightarrow \infty} 0$ in $\mathbb{C}^{2} \times \tilde{H}$.

We can in fact prove a stronger result than this proposition and we can relax the assumption $\nu(\{0\})=0$. Indeed we have

Theorem 3.7. Let $\nu$ be a positive non-null measure satisfying (20). Let $p \geq 1$. Let $u_{e} \in L^{p}([0,+\infty), \mathrm{d} t)$ be such that $u_{e}(t)=0$ for sufficiently large time.

Then we have the asymptotic stability property, i.e. for all $\left(\theta_{0}, \omega_{0}\right) \in \mathbb{C}^{2}$, the solution of (18) in closed-loop with (21), with initial condition $\left(\theta_{0}, \omega_{0}, 0\right)$, satisfies

$$
\tilde{\mathcal{E}}(\theta, \dot{\theta}, \tilde{\Phi})(t) \rightarrow_{t \rightarrow \infty} 0
$$

or equivalently $(\theta, \dot{\theta}, \tilde{\Phi})(t) \rightarrow_{t \rightarrow \infty} 0$ in $\mathbb{C}^{2} \times \tilde{H}$.

Proof. In view of Proposition 3.6 we can restrict ourselves to the case $\nu(\{0\})=C>0$.

Let us first study the case where $\nu$ is a dilation of a Dirac measure i.e. $\nu=C \delta_{0}$ where $\delta_{0}$ is the Dirac measure at $\xi=0$. Then, the differential equation (18) in closed-loop with (21) is equivalent to

$$
\left\{\begin{aligned}
\ddot{\theta}+C \dot{\theta}+c \theta & =0, \\
\partial_{t} \tilde{\Phi}+\xi \tilde{\Phi}+\dot{\theta} & =0, \forall \xi \geq 0,
\end{aligned}\right.
$$

which is known to be an asymptotically stable system. Therefore we have Theorem 3.7 in the case of a Dirac measure.

In the rest of the proof we assume that $\nu=C \delta_{0}+\tilde{\nu}$ where $C$ is a strictly positive number and $\tilde{\nu}$ is a positive measure, non null, with

and satisfying

$$
\tilde{\nu}(\{0\})=0,
$$

$$
c_{\tilde{\nu}}:=\int_{0}^{\infty} \frac{1}{1+\xi} \mathrm{d} \tilde{\nu}(\xi)<\infty .
$$

From (18) and (21) we get

$$
\ddot{\theta}+C \dot{\theta}+\mathcal{D}_{\tilde{\nu}}\left(\partial_{t} \theta\right)+c \theta=u_{e}
$$


which is in closed-loop with

$$
\left\{\begin{aligned}
\partial_{t} \tilde{\phi} & =-\xi \tilde{\phi}+u(t) \\
\tilde{\phi}(0) & =0 \\
z(t) & :=\mathcal{D}_{\nu}\left(\partial_{t} \theta\right)=\int_{0}^{\infty} \partial_{t} \tilde{\phi}(\xi, t) \mathrm{d} \nu(\xi) \\
u(t) & :=\dot{\theta}(t)
\end{aligned}\right.
$$

The differential equations (26) and (27) are equivalent to

$$
\partial_{t}\left(\begin{array}{c}
\theta \\
\omega \\
\tilde{\Phi}
\end{array}\right)=\hat{\mathcal{A}}\left(\begin{array}{c}
\theta \\
\omega \\
\tilde{\Phi}
\end{array}\right)+\tilde{\mathcal{B}} u_{e}:=\left(\begin{array}{c}
\omega \\
\left.-c \theta-C \omega-\int_{0}^{\infty}(\omega-\xi \tilde{\Phi}) \mathrm{d} \tilde{\nu}\right) \\
\omega-\xi \tilde{\Phi}
\end{array}\right)+\left(\begin{array}{c}
0 \\
u_{e} \\
0
\end{array}\right)
$$

with $\hat{\mathcal{A}}: \mathcal{D}(\hat{\mathcal{A}}) \rightarrow \mathbb{C}^{2} \times \tilde{H}$ and $\tilde{\mathcal{B}}: \mathbb{C} \rightarrow \mathbb{C}^{2} \times \tilde{H}$ two suitable linear operators and

$$
\mathcal{D}(\hat{\mathcal{A}})=\mathcal{D}(\tilde{\mathcal{A}})
$$

Since $\hat{A}(\theta, \omega, \tilde{\phi})^{\prime}=\tilde{\mathcal{A}}(\theta, \omega, \tilde{\phi})^{\prime}-C(0, \omega, 0)^{\prime}$ with $C>0$, and since $-\tilde{\mathcal{A}}$ is monotone, the operator $-\hat{\mathcal{A}}$ is also monotone.

We have now to prove the maximality of the operator $-\hat{\mathcal{A}}$.

Lemma 3.8. The operator $-\hat{\mathcal{A}}$ is maximal.

Proof. This proof is a special case of Proposition A.4 with $\check{C}=C$ and $\lambda=1$.

The operator $\hat{\mathcal{A}}$ generates a contraction semigroup and we can claim a result of existence and uniqueness of weak solutions of (28) with initial condition

$$
\theta(0)=\theta_{0}, \quad \omega(0)=\omega_{0}, \quad \tilde{\Phi}(\xi, 0)=0, \forall \xi \in[0,+\infty)
$$

Let us prove that all conditions to apply [1], Stability theorem, are fulfilled. To do this we need to prove the following:

Lemma 3.9. We have

$$
\sigma(\hat{\mathcal{A}}) \cap\{i \alpha, \alpha \in \mathbb{R}, \alpha \neq 0\}=\emptyset .
$$

Proof. This proof is a special case of Proposition A.4 with $\check{C}=C$, and $\lambda=i \alpha, \alpha \in \mathbb{R}, \alpha \neq 0$.

Thanks to Lemma $3.9, \sigma(\hat{\mathcal{A}}) \cap i \mathbb{R}$ is at most countable as required by the necessary conditions of [1], Stability theorem.

Let us now prove that $\operatorname{Ker}(\hat{\mathcal{A}})$ is reduced to the origin.

Lemma 3.10. $\lambda=0$ is not an eigenvalue of $\hat{\mathcal{A}}$.

Proof of Lemma 3.10. From $(28)$ we get that $(\theta, \omega, \tilde{\phi}) \in \operatorname{Ker}(\hat{\mathcal{A}})$ if and only if

$$
\left\{\begin{aligned}
\omega & =0, \\
c \theta+(i \alpha+C) \omega+\int_{0}^{\infty} \xi \tilde{\phi} \mathrm{d} \tilde{\nu} & =0, \\
\omega-\xi \tilde{\phi} & =0, \text { for } \tilde{\nu} \text {-almost every } \xi \text { in }[0, \infty),
\end{aligned}\right.
$$


which is equivalent to

$$
\left\{\begin{aligned}
\omega & =0, \\
\theta & =0, \\
\tilde{\phi} & =0, \text { for } \tilde{\nu} \text {-almost every } \xi \text { in }[0, \infty),
\end{aligned}\right.
$$

since $\tilde{\nu}(\{0\})=0$. Thus $\lambda=0$ is not an eigenvalue of $\hat{\mathcal{A}}$.

We conclude the proof of Theorem 3.7 by using [1], Stability theorem, and Lemmas 3.9 and 3.10.

Note finally that we can state an analogous result for a mixing of the standard diffusive operator (such as the fractional integral) and the extended diffusive operator (such as the fractional derivative). Let us state our result for such a system in the final section.

\section{An oscillator COUPled With Diffusive systems of Both types}

In this section we study an oscillator damped by two pseudo-differential operators, one with a standard diffusive representation, the other with an extended diffusive representation. Under some properties on the measures, we state the asymptotic property of this system. To state our result, we use Theorems 2.6 and 3.7.

We study the following second-order mono-dimensional linear differential equation:

$$
\ddot{\theta}+a\left(\frac{\mathrm{d}}{\mathrm{d} t} \mathcal{D}_{\nu}\right)(\dot{\theta})+b \mathcal{D}_{\mu}(\dot{\theta})+c \theta=u_{e}(t)
$$

where $\theta:[0,+\infty) \rightarrow \mathbb{C}$ is an unknown function, $\mathcal{D}_{\mu}$ and $\mathcal{D}_{\nu}$ are two diffusive pseudo-differential operators with measures $\mu$ and $\nu$ respectively. Let us assume also that $a, b$ and $c$ are three non-negative real numbers, with $a b \neq 0$, and $u_{e}$ is an external input vanishing for sufficiently large time.

We assume moreover that the measures $\mu$ and $\nu$ are positive, non-null and satisfy (7) and (20).

Let us consider (8) and (21), the realizations of the operators $\mathcal{D}_{\mu}$ and $\frac{\mathrm{d}}{\mathrm{d} t} \mathcal{D}_{\nu}$.

We can rewrite (30) as the sum of two oscillators, one in closed-loop with (8), the other one in closed-loop with (21). Due to Theorems 2.6 and 3.7, we have

Theorem 4.1. Let $\mu$ be a positive non-null measure satisfying (7) and such that $\mu(\{0\})=0$ and $\nu$ be a positive non-null measure satisfying (20). Let $p \geq 1$. Let $u_{e} \in L^{p}([0,+\infty)$, $\mathrm{d} t)$ be such that $u_{e}(t)=0$ for sufficiently large time.

Then, we have the asymptotic stability property, i.e. for all $\theta_{0}$ and $\omega_{0}$, the solution of (30) in closed-loop with (8) and (21), with initial condition $\left(\theta_{0}, \omega_{0}, 0,0\right)$ at $t=0$, satisfies

$$
\mathcal{E}(\theta, \dot{\theta}, \Phi)(t)+\tilde{\mathcal{E}}(\theta, \dot{\theta}, \tilde{\Phi})(t) \rightarrow_{t \rightarrow \infty} 0,
$$

or equivalently $(\theta, \dot{\theta}, \Phi, \tilde{\Phi})(t) \rightarrow_{t \rightarrow \infty} 0$ in $\mathbb{C}^{2} \times H \times \tilde{H}$.

Remark 4.2. Note that, thanks to [1], Stability theorem, we prove in fact that the bounded $\mathcal{C}^{0}$-semigroup corresponding to $(2)$ is asymptotically stable which means that for all initial conditions $\left(\theta_{0}, \omega_{0}, \phi_{0}, \tilde{\phi}_{0}\right) \in \mathbb{C}^{2} \times$ $H \times \tilde{H}$, we have

$$
(\theta, \dot{\theta}, \Phi, \tilde{\Phi})(t) \rightarrow_{t \rightarrow \infty} 0 \text { in } \mathbb{C}^{2} \times H \times \tilde{H} .
$$

Now only in the case $\left(\phi_{0}, \tilde{\phi}_{0}\right)=(0,0)$ do we have the input-ouput equivalence of the realizations (2) with (30). 


\section{Conclusion}

In this paper we study a classical oscillator coupled with two causal pseudo-differential operators of diffusive type: the first is of standard type, such as fractional integrals, whereas the second is of extended type, such as fractional derivatives. The well-posedness of the global system is proved by showing a maximally monotone property. The main result concerns the asymptotic stability property of the global system, for which sufficient conditions are derived in terms of the diffusive measures. Due to the lack of compactness of the diffusive part of the problem, LaSalle's invariance principle cannot be used; but the study of the spectrum of the generator of the associated semigroup allows to apply Arendt-Batty theorem. Moreover, illustrative examples are presented with different qualitative behaviours; since the asymptotic stability does not hold in these cases, it shows the sharpness of the main stability result.

\section{Appendix A. Two TeChnicAl RESUlts}

The aim of this section is to prove two technical results, namely Propositions A.2 and A.4, we use thoughout the paper.

First, let us note that

$$
\frac{1}{|\lambda+\xi|} \leq \max \left(1, \frac{1}{|\lambda|}\right) \frac{\sqrt{2}}{1+\xi}
$$

for all $\lambda \in \mathbb{C}$ such that $\Re(\lambda) \geq 0$ and $\lambda \neq 0$ and for all $\xi>0$.

Given $p$ and $q$ two integers and $f: \mathbb{R}_{+} \rightarrow \mathbb{C}$, we denote

$$
c_{\mu}^{p, q}(f)=\int_{0}^{\infty} \frac{(1+\xi)^{p}}{|\lambda+\xi|^{q}}|f|^{2} \mathrm{~d} \mu(\xi),
$$

and

$$
d_{\mu}^{p, q}(f)=\int_{0}^{\infty} \frac{(1+\xi)^{p}}{|\lambda+\xi|^{q}}|f| \mathrm{d} \mu(\xi)
$$

Lemma A.1. For all $k, p$ and $q \in \mathbb{N}$ with $p-q \leq k, f \in H^{k}$ and for all $\lambda \in\{\Re(\lambda) \geq 0\} \backslash\{0\}$, we have:

$$
c_{\mu}^{p, q}(f) \leq\left(\sqrt{2} \max \left(1, \frac{1}{|\lambda|}\right)\right)^{q}\|f\|_{H^{k}}^{2} .
$$

For all $k, p$ and $q \in \mathbb{N}$ with $p-q \leq \frac{k-1}{2}, f \in H^{k}$ and for all $\lambda \in\{\Re(\lambda) \geq 0\} \backslash\{0\}$, we have:

$$
d_{\mu}^{p, q}(f) \leq\left(\sqrt{2} \max \left(1, \frac{1}{|\lambda|}\right)\right)^{q} \sqrt{c_{\mu}}\|f\|_{H^{k}}
$$

Proof. By using (31) and $p-q-k \leq 0$, we have

$$
\begin{aligned}
c_{\mu}^{p, q}(f) & \leq\left(\sqrt{2} \max \left(1, \frac{1}{|\lambda|}\right)\right)^{q} \int_{0}^{\infty}(1+\xi)^{p-q-k}(1+\xi)^{k}|f|^{2} \mathrm{~d} \mu(\xi), \\
& \leq\left(\sqrt{2} \max \left(1, \frac{1}{|\lambda|}\right)\right)^{q} \int_{0}^{\infty}(1+\xi)^{k}|f|^{2} \mathrm{~d} \mu(\xi),
\end{aligned}
$$


which is (32). By using the inequalities (31), $p-q-\frac{k-1}{2} \leq 0,(7)$ and the Cauchy-Schwarz's inequality

$$
\begin{aligned}
d_{\mu}^{p, q}(f) & \leq\left(\sqrt{2} \max \left(1, \frac{1}{|\lambda|}\right)\right)^{q} \int_{0}^{\infty}(1+\xi)^{p-q-\frac{k-1}{2}} \frac{1}{\sqrt{1+\xi}}(1+\xi)^{\frac{k}{2}}|f| \mathrm{d} \mu(\xi) \\
& \leq\left(\sqrt{2} \max \left(1, \frac{1}{|\lambda|}\right)\right)^{q} \int_{0}^{\infty} \frac{1}{\sqrt{1+\xi}}(1+\xi)^{\frac{k}{2}}|f| \mathrm{d} \mu(\xi) \\
& \leq\left(\sqrt{2} \max \left(1, \frac{1}{|\lambda|}\right)\right)^{q} \sqrt{\int_{0}^{\infty} \frac{1}{1+\xi} \mathrm{d} \mu(\xi)}, \sqrt{\int_{0}^{\infty}(1+\xi)^{k}|f|^{2} \mathrm{~d} \mu(\xi)}
\end{aligned}
$$

which is (33). This concludes the proof of Lemma A.1.

With this result at hand, let us prove now the following

Proposition A.2. For all $\lambda \in\{\Re(\lambda) \geq 0\} \backslash\{0\},(\lambda I-\mathcal{A})^{-1}$ is a bounded operator from $\mathbb{C}^{2} \times H$ to $\mathbb{C}^{2} \times H$.

Proof. Let us first note that, using (33) with $p=0, q=1$ and $k=0$, we have

$$
\left(\int_{0}^{\infty}\left|\frac{f}{\lambda+\xi}\right| \mathrm{d} \mu(\xi)\right)^{2} \leq 2 c_{\mu} \max \left(1, \frac{1}{|\lambda|^{2}}\right)\|f\|_{H}^{2}
$$

for all $f$ in $H$. Moreover, using (32) with $p=0, q=1$ and $k=-1$ and $f=1$ we have

$$
\int_{0}^{\infty}\left|\frac{1}{\lambda+\xi}\right| \mathrm{d} \mu(\xi) \leq \sqrt{2} \max \left(1, \frac{1}{|\lambda|}\right) c_{\mu} .
$$

Thus we have $(\lambda I-\mathcal{A})(\theta, \omega, \phi)^{\prime}=(x, y, f)^{\prime}$, with $(\theta, \omega, \phi) \in \mathcal{D}(\mathcal{A})$ and $(x, y, f) \in \mathbb{C}^{2} \times H$ if and only if, for all $\xi \geq 0$, the following holds

$$
\begin{aligned}
\lambda \theta-\omega & =x, \\
c \theta+\left(\lambda+\int_{0}^{\infty} \frac{\mathrm{d} \mu(\xi)}{\lambda+\xi}\right) \omega & =y-\int_{0}^{\infty} \frac{1}{\lambda+\xi} f \mathrm{~d} \mu(\xi), \\
\phi & =\frac{f+\omega}{\lambda+\xi}
\end{aligned}
$$

Let us first study the linear operator $(\theta, \omega) \mapsto(x, y)$ defined by (36)-(37). Due to (34), the second member of the equations (36)-(37) is continuous. Moreover, it is continuously invertible if and only if

$$
\Delta(\lambda) \neq 0
$$

where

$$
\begin{aligned}
\Delta(\lambda) & :=\lambda^{2}+\lambda \int_{0}^{\infty} \frac{\mathrm{d} \mu(\xi)}{\lambda+\xi}+c \\
& =\lambda^{2}+|\lambda|^{2} \int_{0}^{\infty} \frac{\mathrm{d} \mu(\xi)}{|\lambda+\xi|^{2}}+\lambda \int_{0}^{\infty} \frac{\xi}{|\lambda+\xi|^{2}} \mathrm{~d} \mu(\xi)+c
\end{aligned}
$$


Two cases may been inspected:

- for $\lambda>0$, we have $\Delta(\lambda) \geq c>0$;

- for $\Im(\lambda) \neq 0$ and $\Re(\lambda) \geq 0$, inspecting the imaginary part of $\Delta(\lambda)$ gives

$$
\Im(\Delta(\lambda))=\Im(\lambda)\left(2 \Re(\lambda)+\int_{0}^{\infty} \frac{\xi}{|\lambda+\xi|^{2}} \mathrm{~d} \mu(\xi)\right) \neq 0
$$

where $\Im$ denotes the imaginary part of a complex number.

Thus $(\theta, \omega) \mapsto(x, y)$ is continuously invertible.

To achieve the proof of the invertibility and the continuity of the operator $(\lambda I-\mathcal{A})^{-1}$, with $\lambda \in\{\Re(\lambda) \geq$ $0\} \backslash\{0\}$, it remains to check that

- the function $\phi$ defined by (38) lies in $V$. To do this, let us note that (35) implies that $\xi \mapsto \frac{1}{\lambda+\xi} \in V$. Moreover, using (32) with $p=1, q=2$ and $k=0$, we have

$$
\int_{0}^{\infty}(1+\xi) \frac{|f|^{2}}{|\lambda+\xi|^{2}} \mathrm{~d} \mu(\xi)<+\infty
$$

- the function $\xi \mapsto \xi \phi-\omega$ lies in $H$. Note that this function can been restated as

$$
\xi \phi-\omega=\frac{\xi}{\lambda+\xi} f-\frac{\lambda}{\lambda+\xi} \omega .
$$

We have already remarked in the previous case that $\xi \mapsto \frac{1}{\lambda+\xi} \in V \subset H$. Moreover,

$$
\int_{0}^{\infty} \frac{\xi^{2}}{|\lambda+\xi|^{2}}|f|^{2} \mathrm{~d} \mu(\xi) \leq \int_{0}^{\infty}|f|^{2} \mathrm{~d} \mu<+\infty
$$

since $\Re(\lambda) \geq 0$. Therefore $\xi \mapsto \xi \phi-\omega$ lies in $H$.

- To conclude, let us prove the continuity of the operator $(x, y, f) \mapsto \phi \in V$. By using (32) with $p=1$, $q=2, k=0$ and $f=f$ and with $p=1, q=2, k=-1$ and $f=1$ we have:

$$
\begin{aligned}
\|\phi\|_{V}^{2} & =\int_{0}^{\infty}(1+\xi) \frac{|f+\omega|^{2}}{|\lambda+\xi|^{2}} \mathrm{~d} \mu(\xi) \\
& \leq 2 \int_{0}^{\infty} \frac{1+\xi}{|\lambda+\xi|^{2}}\left(|f|^{2}+\omega^{2}\right) \mathrm{d} \mu(\xi) \\
& \leq 4 \max \left(1, \frac{1}{|\lambda|^{2}}\right)\left(\|f\|_{H}^{2}+c_{\mu} \omega^{2}\right), \\
& \leq 4 \max \left(1, \frac{1}{|\lambda|^{2}}\right)\left(1+c_{\mu} M\right)\left(|x|^{2}+|y|^{2}+\|f\|_{H}^{2}\right),
\end{aligned}
$$

where $M$ is a positive constant depending only on the linear operator $(x, y, f) \mapsto \omega$ defined by $(36)-(37)$. This achieves the proof of Proposition A.2.

Given $p$ and $q$ two integers and $\tilde{f}: \mathbb{R}_{+} \rightarrow \mathbb{C}$, we denote

$$
c_{\nu}^{p, q}(\tilde{f})=\int_{0}^{\infty} \xi \frac{(1+\xi)^{p}}{|\lambda+\xi|^{q}}|\tilde{f}|^{2} \mathrm{~d} \nu(\xi),
$$

and

$$
d_{\nu}^{p, q}(\tilde{f})=\int_{0}^{\infty} \xi \frac{(1+\xi)^{p}}{|\lambda+\xi|^{q}}|\tilde{f}| \mathrm{d} \nu(\xi)
$$


Lemma A.3. For all $p$ and $q \in \mathbb{N}$ with $p-q \leq 0, f \in \tilde{H}$ and for all $\lambda \in\{\Re(\lambda) \geq 0\} \backslash\{0\}$, we have:

$$
c_{\nu}^{p, q}(\tilde{f}) \leq\left(\sqrt{2} \max \left(1, \frac{1}{|\lambda|}\right)\right)^{q}\|\tilde{f}\|_{\tilde{H}}^{2}
$$

For all $k, p$ and $q \in \mathbb{N}$ with $p-q \leq-1, \tilde{f} \in \tilde{H}$ and for all $\lambda \in\{\Re(\lambda) \geq 0\} \backslash\{0\}$, we have:

$$
d_{\nu}^{p, q}(\tilde{f}) \leq\left(\sqrt{2} \max \left(1, \frac{1}{|\lambda|}\right)\right)^{q} \sqrt{c_{\nu}}\|\tilde{f}\|_{\tilde{H}}
$$

Proof. By using (31) and $p-q \leq 0$, we have

$$
\begin{aligned}
c_{\nu}^{p, q}(\tilde{f}) & \leq\left(\sqrt{2} \max \left(1, \frac{1}{|\lambda|}\right)\right)^{q} \int_{0}^{\infty} \xi(1+\xi)^{p-q}|\tilde{f}|^{2} \mathrm{~d} \nu(\xi), \\
& \leq\left(\sqrt{2} \max \left(1, \frac{1}{|\lambda|}\right)\right)^{q} \int_{0}^{\infty} \xi|\tilde{f}|^{2} \mathrm{~d} \nu(\xi),
\end{aligned}
$$

which is (39). By using the inequalities $(31), p-q+1 \leq 0,(20)$ and the Cauchy-Schwarz's inequality

$$
\begin{aligned}
d_{\nu}^{p, q}(\tilde{f}) & \leq\left(\sqrt{2} \max \left(1, \frac{1}{|\lambda|}\right)\right)^{q} \int_{0}^{\infty} \xi(1+\xi)^{p-q+1} \frac{1}{\sqrt{1+\xi}} \frac{1}{\sqrt{1+\xi}}|\tilde{f}| \mathrm{d} \nu(\xi), \\
& \leq\left(\sqrt{2} \max \left(1, \frac{1}{|\lambda|}\right)\right)^{q} \int_{0}^{\infty} \frac{\sqrt{\xi}}{\sqrt{1+\xi}} \frac{1}{\sqrt{1+\xi}} \sqrt{\xi}|\tilde{f}| \mathrm{d} \nu(\xi), \\
& \leq\left(\sqrt{2} \max \left(1, \frac{1}{|\lambda|}\right)\right)^{q} \sqrt{\int_{0}^{\infty} \frac{1}{1+\xi} \mathrm{d} \nu(\xi)}, \sqrt{\int_{0}^{\infty} \xi|\tilde{f}|^{2} \mathrm{~d} \nu(\xi),}
\end{aligned}
$$

which is (40). This concludes the proof of Lemma A.3.

Let $\check{C} \geq 0$ and $\check{\mathcal{A}}$ defined on $\mathcal{D}(\tilde{\mathcal{A}})$, by $\check{\mathcal{A}}(\theta, \omega, \tilde{\phi})^{\prime}=\tilde{\mathcal{A}}(\theta, \omega, \tilde{\phi})^{\prime}-(0, \check{C} \omega, 0)^{\prime}$. Let us now prove an analogous result of Proposition A.2 on $\check{\mathcal{A}}$.

Proposition A.4. For all $\lambda \in\{\Re(\lambda) \geq 0\} \backslash\{0\},(\lambda I-\check{\mathcal{A}})^{-1}$ is a bounded operator from $\mathbb{C}^{2} \times \tilde{H}$ to $\mathbb{C}^{2} \times \tilde{H}$.

Proof. Let us first note that, using (40) with $p=0$ and $q=1$, we have

$$
\int_{0}^{\infty}\left|\frac{\xi \tilde{f}}{\lambda+\xi}\right| \mathrm{d} \nu(\xi) \leq \sqrt{2} \max \left(1, \frac{1}{|\lambda|}\right) \sqrt{c_{\nu}}\|\tilde{f}\|_{\tilde{H}}
$$

for all $f$ in $\tilde{H}$. Moreover, using (20) and (31), we have

$$
\int_{0}^{\infty}\left|\frac{1}{\lambda+\xi}\right| \mathrm{d} \nu(\xi) \leq \sqrt{2} \max \left(1, \frac{1}{|\lambda|}\right) c_{\nu} .
$$


Thus we have $(\lambda I-\check{\mathcal{A}})(\theta, \omega, \tilde{\phi})^{\prime}=(x, y, \tilde{f})^{\prime}$, with $(\theta, \omega, \tilde{\phi}) \in \mathcal{D}(\tilde{\mathcal{A}})$ and $(x, y, \tilde{f}) \in \mathbb{C}^{2} \times \tilde{H}$ if and only if, for all $\xi \geq 0$, the following holds

$$
\begin{aligned}
\lambda \theta-\omega & =x, \\
c \theta+\left(\check{C}+\lambda+\int_{0}^{\infty} \frac{\lambda \mathrm{d} \nu(\xi)}{\lambda+\xi}\right) \omega & =y-\int_{0}^{\infty} \frac{\xi}{\lambda+\xi} \tilde{f} \mathrm{~d} \nu(\xi), \\
\tilde{\phi} & =\frac{\tilde{f}+\omega}{\lambda+\xi} .
\end{aligned}
$$

Let us first study the linear operator $(\theta, \omega) \mapsto(x, y)$ defined by (43)-(44). Due to (41), the second member of the equations (43)-(44) is continuous. Moreover, it is continuously invertible if and only if

$$
\tilde{\Delta}(\lambda) \neq 0
$$

where

$$
\begin{aligned}
\tilde{\Delta}(\lambda) & :=\lambda \check{C}+\lambda^{2}+\lambda^{2} \int_{0}^{\infty} \frac{\mathrm{d} \nu(\xi)}{\lambda+\xi}+c \\
& =\lambda \check{C}+\lambda^{2}+\lambda|\lambda|^{2} \int_{0}^{\infty} \frac{\mathrm{d} \nu(\xi)}{|\lambda+\xi|^{2}}+\lambda^{2} \int_{0}^{\infty} \frac{\xi}{|\lambda+\xi|^{2}} \mathrm{~d} \nu(\xi)+c .
\end{aligned}
$$

Two cases may been inspected:

- for $\lambda>0$, we have $\Delta(\lambda) \geq c>0$;

- for $\Im(\lambda) \neq 0$ and $\Re(\lambda) \geq 0$, inspecting the imaginary part of $\Delta(\lambda)$ gives

$$
\Im(\Delta(\lambda))=\Im(\lambda)\left(\check{C}+2 \Re(\lambda)+2 \Re(\lambda) \int_{0}^{\infty} \frac{\xi}{|\lambda+\xi|^{2}} \mathrm{~d} \nu(\xi)+|\lambda|^{2} \int_{0}^{\infty} \frac{1}{|\lambda+\xi|^{2}} \mathrm{~d} \nu(\xi)\right) \neq 0 .
$$

Thus $(\theta, \omega) \mapsto(x, y)$ is continuously invertible.

To achieve the proof of the invertibility and the continuity of the operator $(\lambda I-\check{\mathcal{A}})^{-1}$, with $\lambda \in\{\Re(\lambda) \geq$ $0\} \backslash\{0\}$, it remains to check that

- the function $\tilde{\phi}$ defined by (45) lies in $\tilde{H}$. To do this, let us note that (42) implies that $\xi \mapsto \frac{1}{\lambda+\xi} \in V$. Moreover, using (39) with $p=0$ and $q=2$, we have

$$
\int_{0}^{\infty} \xi \frac{|\tilde{f}|^{2}}{|\lambda+\xi|^{2}} \mathrm{~d} \nu(\xi) \leq 2 \max \left(1, \frac{1}{|\lambda|^{2}}\right)\|\tilde{f}\|_{\tilde{H}}^{2}
$$

- the function $\xi \mapsto \xi \phi-\omega$ lies in $V$. Note that this function can been restated as

$$
\xi \phi-\omega=\frac{\xi}{\lambda+\xi} \tilde{f}-\frac{\lambda}{\lambda+\xi} \omega .
$$

We have already remarked in the previous case that $\xi \mapsto \frac{1}{\lambda+\xi} \in V$. Moreover, using (39) with $p=q=2$, we have

$$
\int_{0}^{\infty} \frac{(1+\xi) \xi^{2}}{|\lambda+\xi|^{2}}|f|^{2} \mathrm{~d} \nu(\xi) \leq \int_{0}^{\infty} \frac{(1+\xi)^{2}}{|\lambda+\xi|^{2}} \xi|f|^{2} \mathrm{~d} \nu(\xi) \leq 2 \max \left(1, \frac{1}{|\lambda|^{2}}\right)\|f\|_{\tilde{H}}^{2},
$$

since $\Re(\lambda) \geq 0$. Therefore $\xi \mapsto \xi \phi-\omega$ lies in $V$. 
- To conclude, let us prove the continuity of the operator $(x, y, f) \mapsto \phi \in \tilde{H}$. By using (39) with $p=0$, $q=2$ and $\tilde{f}=\tilde{f}$, and with $p=1, q=2$ and $\tilde{f}(\xi)=\frac{1}{1+\xi}$, we have

$$
\begin{aligned}
\|\phi\|_{\tilde{H}}^{2} & =\int_{0}^{\infty} \xi \frac{|\tilde{f}+\omega|^{2}}{|\lambda+\xi|^{2}} \mathrm{~d} \nu(\xi), \\
& \leq 2 \int_{0}^{\infty} \frac{\xi}{|\lambda+\xi|^{2}}\left(|\tilde{f}|^{2}+\omega^{2}\right) \mathrm{d} \nu(\xi), \\
& \leq 4 \max \left(1, \frac{1}{|\lambda|^{2}}\right)\left(\|\tilde{f}\|_{\tilde{H}}^{2}+c_{\nu} \omega^{2}\right), \\
& \leq 4 \max \left(1, \frac{1}{|\lambda|^{2}}\right)\left(1+c_{\nu} \tilde{M}\right)\left(|x|^{2}+|y|^{2}+\|\tilde{f}\|_{\tilde{H}}^{2}\right),
\end{aligned}
$$

where $\tilde{M}$ is a positive constant depending only on the linear operator $(x, y, \tilde{f}) \mapsto \omega$ defined by (43)-(44). This achieves the proof of Proposition A.4.

\section{Appendix B. Proof of Remark 2.8}

The aim of this section if to prove Remark 2.8 by studying two examples.

\section{B.1. First example: $\mu=C_{0} \delta_{0}$}

Assume first that $\mu=C_{0} \delta_{0}$, where $C_{0}$ is a strictly positive real number and $\delta_{0}$ is the Dirac measure at $\xi=0$. Then (5) and (8) imply

$$
\begin{gathered}
\ddot{\theta}+y+c \theta=0, \\
y(t)=C_{0} \Phi(0, t)=C_{0}\left(\theta(t)-\theta_{0}\right),
\end{gathered}
$$

thus

$$
\begin{gathered}
\ddot{\theta}+\left(C_{0}+c\right) \theta=C_{0} \theta_{0}, \\
y(t)=C_{0}\left(\theta(t)-\theta_{0}\right),
\end{gathered}
$$

which, of course, is not an asymptotically stable system, since

$$
\theta(t)=\frac{C_{0}}{C_{0}+c} \theta_{0}+\frac{c \theta_{0}}{C_{0}+c} \cos \left(\sqrt{C_{0}+c} t\right)+\frac{\omega_{0}}{\sqrt{C_{0}+c}} \sin \left(\sqrt{C_{0}+c} t\right)
$$

for the initial condition $\left(\theta_{0}, \omega_{0}\right)$.

B.2. Second example: $\mu=C_{0} \delta_{0}+C_{1} \mu_{\alpha}$

Assume now that

$$
\mu=C_{0} \delta_{0}+C_{1} \mu_{\alpha}
$$

where the non-null positive measure $\mu_{\alpha}$ has a density $\mathrm{d} \mu_{\alpha}(\xi)=\frac{\sin (\pi \alpha)}{\pi} \frac{\mathrm{d} \xi}{\xi^{\alpha}}$, for all $\xi>0$, for $0<\alpha<1$ and $C_{0}$, $C_{1}>0$. We have (see e.g. [18])

$$
\int_{0}^{\infty} \frac{\mu_{\alpha}(\xi)}{s+\xi} \mathrm{d} \xi=\frac{1}{s^{\alpha}}, \forall s, \Re(s)>0
$$

and thus $\mathcal{D}_{\mu_{\alpha}}$ can be seen as $I^{\alpha}$ the causal fractional integral of order $\alpha$. Note that $\mu_{\alpha}(\{0\})=0$ and fulfills (7). 
System (5)-(8) is not asymptotically stable either. Indeed the $\theta$-component of the solution does not tend to 0 either:

Proposition B.1. Let $0<\alpha<1$ and $C_{0}, C_{1}>0$. Let $p \geq 1$. Let $u_{e} \in L^{p}([0,+\infty), \mathrm{d} t)$ be such that $u_{e}(t)=0$ for sufficiently large time.

Then the solution of (5) in closed-loop with (8), with initial condition $\left(\theta_{0}, \omega_{0}, 0\right)$ at $t=0$, satisfies

$$
(\theta, \dot{\theta})(t) \rightarrow_{t \rightarrow \infty}\left(\frac{C_{0} \theta_{0}}{c+C_{0}}, 0\right) .
$$

Proof. Let $u_{e}$ in $L^{p}([0,+\infty), \mathrm{d} t)$ be such that

$$
u_{e}(t)=0, \forall t \geq T_{e},
$$

where $T_{e}>0$.

To prove Proposition B.1, let us note first that System (5)-(8) implies

$$
\begin{aligned}
y & =\int_{0}^{\infty} \Phi \mathrm{d} \mu, \\
& =C_{0} \Phi(\xi=0)+C_{1} \int_{0}^{\infty} \Phi \mathrm{d} \mu_{\alpha}, \\
& =C_{0}\left(\theta(t)-\theta_{0}\right)+y_{\alpha},
\end{aligned}
$$

where

Therefore

$$
y_{\alpha}:=C_{1} \int_{0}^{\infty} \Phi \mathrm{d} \mu_{\alpha}
$$

$$
\left\{\begin{aligned}
\ddot{\theta}+y_{\alpha}+\left(c+C_{0}\right) \theta & =C_{0} \theta_{0}+u_{e}, \\
\partial_{t} \Phi & =-\xi \Phi+u, \\
u & =\dot{\theta}, \\
y_{\alpha} & =C_{1} \int_{0}^{\infty} \Phi \mathrm{d} \mu_{\alpha}, \\
\theta(0) & =\theta_{0}, \\
\dot{\theta}(0) & =\omega_{0}, \\
\Phi(., t) & =0,
\end{aligned}\right.
$$

for the initial conditions $\left(\theta_{0}, \omega_{0}, 0\right) \in \mathcal{D}(\mathcal{A})$. Thanks to $(48)$, taking the Laplace transform of $(50)$, we have

$$
s^{2} \hat{\theta}-s \theta_{0}-\omega_{0}+\frac{C_{1}}{s^{\alpha}}\left(s \hat{\theta}-\theta_{0}\right)+\left(c+C_{0}\right) \hat{\theta}=\frac{C_{0} \theta_{0}}{s}+\hat{u}_{e}
$$

where $\hat{\theta}: \mathbb{C}_{+} \rightarrow \mathbb{C}$ is the Laplace transform of $\theta, \hat{u}_{e}: \mathbb{C}_{+} \rightarrow \mathbb{C}$ is the Laplace transform of $u_{e}$ and $\mathbb{C}_{+}=$ $\{s, \Re(s)>0\}$.

Let us prove that we have

$$
s^{2}+C_{1} s^{1-\alpha}+c+C_{0} \neq 0, \forall s \in \mathbb{C}_{+}
$$

Indeed

- it is obvious on $\mathbb{R}_{+}$;

- taking the imaginary part of $s^{2}+C_{1} s^{1-\alpha}+c+C_{0}$, for $s=\rho e^{i \gamma} \in \mathbb{C}_{+} \backslash \mathbb{R}_{+}$, yields $\rho^{2} \sin (2 \gamma)+$ $C_{1} \rho^{1-\alpha} \sin ((1-\alpha) \gamma)$, which has exactly the same sign as $\gamma \in\left[-\frac{\pi}{2}, \frac{\pi}{2}\right] \backslash\{0\}$, and thus not zero. 
Thanks to (51) and (52), we have

$$
s \hat{\theta}(s)=\frac{C_{0} \theta_{0}+C_{1} s^{1-\alpha} \theta_{0}+s \omega_{0}+s^{2} \theta_{0}}{c+C_{0}+C_{1} s^{1-\alpha}+s^{2}}+\frac{s \hat{u}_{e}(s)}{c+C_{0}+C_{1} s^{1-\alpha}+s^{2}} .
$$

Thanks to $(49), \hat{u}_{e}(0)=\int_{0}^{T_{e}} u_{e}(t) \mathrm{d} t$ and thus $\lim _{s \rightarrow 0, s \in \mathbb{C}_{+}} s \hat{u}_{e}(s)=0$. Moreover, since $0<\alpha<1$ and thanks to (53), we have

$$
\begin{aligned}
\lim _{t \rightarrow+\infty} \theta(t) & =\lim _{s \rightarrow 0, s \in \mathbb{C}_{+}} s \hat{\theta}(s), \\
& =\frac{C_{0} \theta_{0}}{c+C_{0}}
\end{aligned}
$$

and

$$
\begin{aligned}
\lim _{t \rightarrow+\infty} \dot{\theta}(t) & =\lim _{s \rightarrow 0,} s\left(s \hat{\theta}(s)-\theta_{0}\right), \\
& =0 .
\end{aligned}
$$

This concludes the proof of Proposition B.1.

Acknowledgements. The authors would like to thank F. Conrad and M. Tucsnak for indicating [1]. The anonymous reviewers have helped enhance the presentation and conciseness of the paper, and are gratefully acknowledged.

\section{REFERENCES}

[1] W. Arendt and C.J.K. Batty, Tauberian theorems and stability of one-parameter semigroups. Trans. Am. Math. Soc. 306 (1988) 837-852.

[2] H. Brezis, Analyse fonctionnelle. Théorie et applications. Masson, Paris (1983).

[3] M. Bruneau, Ph. Herzog, J. Kergomard and J.-D. Polack, General formulation of the dispersion equation in bounded viscothermal fluid, and application to some simple geometries. Wave Motion 11 (1989) 441-451.

[4] T. Cazenave and A. Haraux, An introduction to semilinear evolution equations. Oxford Lecture Series in Mathematics and its Applications 13 (1998).

[5] F. Conrad and M. Pierre, Stabilization of second order evolution equations by unbounded nonlinear feedback. Ann. Inst. Henri Poincaré, Anal. Non Linéaire 11 (1994) 485-515.

[6] R.F. Curtain and H. Zwart, An introduction to infinite-dimensional linear systems theory. Texts Appl. Math. 21 (1995).

[7] G. Dauphin, D. Heleschewitz and D. Matignon, Extended diffusive representations and application to non-standard oscillators, in Proc. of Math. Theory on Network Systems (MTNS), Perpignan, France (2000).

[8] R. Dautray and J.-L. Lions, Mathematical analysis and numerical methods for science and technology, Vol. 5. Springer, New York (1984).

[9] Z.E.A. Fellah, C. Depollier and M. Fellah, Direct and inverse scattering problem in porous material having a rigid frame by fractional calculus based method. J. Sound Vibration 244 (2001) 3659-3666.

[10] H. Haddar, T. Hélie and D. Matignon, A Webster-Lokshin model for waves with viscothermal losses and impedance boundary conditions: strong solutions, in Proc. of Sixth international conference on mathematical and numerical aspects of wave propagation phenomena, Jyväskylä, Finland (2003) 66-71.

[11] Th. Hélie, Unidimensional models of acoustic propagation in axisymmetric waveguides. J. Acoust. Soc. Am. 114 (2003) 26332647.

[12] A.E. Ingham, On Wiener's method in Tauberian theorems, in Proc. London Math. Soc. II 38 (1935) 458-480.

[13] J. Korevaar, On Newman's quick way to the prime number theorem. Math. Intell. 4 (1982) 108-115.

[14] A.A. Lokshin, Wave equation with singular retarded time. Dokl. Akad. Nauk SSSR 240 (1978) 43-46 (in Russian).

[15] A.A. Lokshin and V.E. Rok, Fundamental solutions of the wave equation with retarded time. Dokl. Akad. Nauk SSSR 239 (1978) 1305-1308 (in Russian).

[16] Z.-H. Luo, B.-Z. Guo and O. Morgul, Stability and stabilization of infinite dimensional systems and applications. Comm. Control Engrg. Springer-Verlag, New York (1999).

[17] Yu.I. Lyubich and V.Q. Phóng, Asymptotic stability of linear differential equations in Banach spaces. Stud. Math. 88 (1988) 37-42. 
[18] D. Matignon, Stability properties for generalized fractional differential systems. ESAIM: Proc. 5 (1998) 145-158.

[19] G. Montseny, Diffusive representation of pseudo-differential time-operators. ESAIM: Proc. 5 (1998) $159-175$.

[20] D.J. Newman, Simple analytic proof of the prime number theorem. Am. Math. Mon. 87 (1980) $693-696$.

[21] J.-D. Polack, Time domain solution of Kirchhoff's equation for sound propagation in viscothermal gases: a diffusion process. J. Acoustique 4 (1991) 47-67.

[22] O.J. Staffans, Well-posedness and stabilizability of a viscoelastic equation in energy space. Trans. Am. Math. Soc. 345 (1994) $527-575$.

[23] O.J. Staffans, Passive and conservative continuous-time impedance and scattering systems. Part I: Well-posed systems. Math. Control Sig. Syst. 15 (2002) 291-315.

[24] G. Weiss, O.J. Staffans and M. Tucsnak, Well-posed linear systems - a survey with emphasis on conservative systems. Internat. J. Appl. Math. Comput. Sci. 11 (2001) 7-33.

[25] G. Weiss and M. Tucsnak, How to get a conservative well-posed linear system out of thin air. Part I. Well-posedness and energy balance. ESAIM: COCV 9 (2003) 247-273. 